No, this is not a chronicle of optical fiber development for the communications industry although it does concern communications. Neither are we about to expound on the search for a new breed of elementary particle with yet another set of evocative quantum states (e.g., the opaque quark, transparent quark, and translucent or fuzzy quark). We do want to describe a quest, however, for an equally elusive state. We can call it the state of meetings grace. Or, perhaps more to the point, meeting facilities made in heaven.

What do you recall from recent conferences you've attended about the facilities, either specifically or generally? You probably recall a few less-than-flattering aspects. Perhaps you also recall some positive points, remarkable only because they were the exception to the rule. It may take some effort to dredge up details because, if you're like many, repeated exposure to the effect of facilities on one's senses numbs them, leaving us oblivious to our surroundings after a time.

Shall we enumerate some of the principal annoyances? The most ubiquitous is noise from a plethora of sources. (We do not include here the chatter from miscreant attendees in your very own room because we treated that phenomenon in an earlier POSTERMINARIES.') Noise of mechanical origin includes the fans on the slide and overhead projectors, particularly noticeable in a small room or if you are seated next to one. There is the whoosh of the powerful heating and air conditioning systems as they try to force hundreds of cubic feet of air per minute through plenums and grates. Even with electronic amplification, a speaker is often hard put to compete with the drone and the switching on and off, especially when vibrating fans and compressors are nearby. Let us also not forget to credit those gorgeous crystal chandeliers that adorn the largest hotel ballrooms and add a tinkling accent to the whoosh.

There is also the decidedly nonmechanical chorus of human hall noise that punctuates proceedings as doors are opened and closed. These are not without their own mechanical grace notes, however. The combination of mid- to highoctave hinge squeaking with low-range clanks of crash bars and latches reminds one of sound effects appropriate to tales of a haunted house.

In that vein, we also contend with hidden corridors running along one side of each big room in the major meeting houses such as hotels and convention

\section{Search for Transparency}

centers. These are gratuitously called service corridors (indeed when eating rather than meeting, it is from them that the food magically appears). During a meeting, they serve to let us hear the clatter of carts being pushed (the ones with the one-wobbly-wheel syndrome are the best) and of tables of the circular variety being rolled to the banquet setup next door. Color is often added by the raucous give and take in the genre of happy service crews everywhere. They are understandably unaware of your proximity. It is, after all, unlikely that the topic with which the speaker in your room is trying to hold your attention would interest them much. We somehow cannot picture even the most conscientious hotel employees silently huddling about the crack in the service entrance door trying desperately to hear the next chapter in the "laser annealing of silicon" saga.

Beside the walls that shield us from the ugly service access and define the boundary between teaming coffee break and seminar in session are the walls between meeting rooms. Perhaps charity dictates that we are afforded the chance, involuntarily of course, to sit within earshot of the wall and hear the speaker next door as an added benefit of attendance. But how can the same be said of the opportunity to vicariously participate in the "rah-rah" sales force meeting designed to enthuse the faithful, working on commission?

Enough about noise. There is more! There are the lights that either can't be turned on or can't be turned off depending on what is most inconvenient at the time for simultaneously seeing a projected micrograph on the screen and seeing the notes in front of your nose. There are the slide projector platforms placed in the aisle or by the door at just the right height and angle to assure that we will be treated to the silhouettes of our colleagues. You know we are all sensitive to this when you see one person after another ducking to avoid the beam as they enter and leave the room even when the projector is off.

There is the overhead projector and screen combination which invariably projects too short an image in one direction or another. The challenge of reading axis labels as the speaker fiddles in vain to prevent truncation cannot be overstated. This phenomenon may go unnoticed on the modern lenticular screens which not only intensify the image but enable glancing illumination from the spotlight in the ceiling to augment the image with a blind- ing glare. No sun, no sand, no surf - just glare.

The point is probably made. A truly transparent meeting facility would not be in the least memorable. It's just that attending the meeting would, for some inexplicable reason, be more of a pleasure than a chore.

It is tempting to start listing the hightech, and admittedly expensive, solutions to all these peevish complaints. Suffice it to note that in concert halls, the air conditioning is absolutely silent. Suffice it to ask, if air locks undersea can keep the water out and light locks in darkrooms can keep the light out, why can't sound locks (without squeaky hinges) keep the sound out where it belongs? In fact if we can see instantaneously what is happening on the other side of the world, why can't we see enough of what's happening in a meeting room to decide beforehand whether we need to clank a latch, let that belch of hall noise in, risk having a slide projected on the side of our head, and pretend we don't notice how many heads turn to see if anyone of consequence has entered the room?*

Call it a quest for meeting nirvana or an unrealistic meeting Utopia, but who among us would not pay a ten times higher registration fee to enjoy a more comfortable chair and a little transparency...

ELTON N. KAUFMANN

1. "Meetings in the Last Row", MRS BULLETIN, 12 (2) (1987) p. 100.

*So many more facility attributes could be mentioned. We had wanted to mention the L-shaped room and well-placed columns, the minuscule clasps on lavaliere mikes and speakers positioned to enhance feedback, thermostats (if any) placed where cumulative body heat is never felt, etc. However, we have been enjoined for two reasons not to overdo. It would be counterproductive timing to emphasize too strongly the down side of meetings as the MRS Fall Meeting extravaganza looms. And, the BULLETIN'S page budget has no provision for the kind of excess upon which we had wanted to embark. Although we reject the former out of hand because MRS topics transfix participants to the point of achieving quasitransparency, we must alas agree that, based on how little of the last page remains, diminishing returns have been reached insofar as the cost of paper and ink. 